\title{
Handgrip Strength and Vitamin D as Predictors of Liver Fibrosis and Malnutrition in Chronic Hepatitis C Patients
}

\author{
Sami A. Gabr $\mathbb{D}^{1,2}$ and Ahmad H. Alghadir $\mathbb{D}^{1}$ \\ ${ }^{1}$ Rehabilitation Research Chair, College of Applied Medical Sciences, King Saud University, Riyadh, Saudi Arabia \\ ${ }^{2}$ Department of Anatomy, Faculty of Medicine, Mansoura University, Egypt \\ Correspondence should be addressed to Sami A. Gabr; dr.samigabr@gmail.com
}

Received 4 October 2020; Revised 15 March 2021; Accepted 22 March 2021; Published 5 April 2021

Academic Editor: Chao Hung Hung

Copyright (C) 2021 Sami A. Gabr and Ahmad H. Alghadir. This is an open access article distributed under the Creative Commons Attribution License, which permits unrestricted use, distribution, and reproduction in any medium, provided the original work is properly cited.

\begin{abstract}
Background. In patients with chronic hepatitis $\mathrm{C}(\mathrm{CHC})$, a negative impact of associated malnutrition on both morbidity and mortality was reported. We aimed to elucidate the efficacy of serum liver fibrosis markers (fibronectin (FN), hydroxyproline (Hyp), and hyaluronic acid (HA)) and their respective indices (HA index, Hyp index, and FN index) and vitamin D status in predicting malnutrition associated with liver fibrosis in $\mathrm{CHC}$ patients and to investigate their association with the value of current clinical malnutrition assessment tools subjective global assessment (SGA), handgrip strength (HGS), and muscle mass scores (SGA, BMI, MAMC, and HGS). Materials and Methods. A cross-sectional study was conducted on 80 patients aged 40-60 years with proven viremia, $\mathrm{HCV}$ antibodies, $\mathrm{HCV}$-RNA positivity, genotype determinations, and established chronic hepatitis $\mathrm{C}$ virus for more than 6 years and 80 control subjects. SGA, HGS, and muscle mass score (MAMC) were estimated in both patients and control subjects. Based on SGA scores, CHC patients were classified into three groups: well nourished ( $n=12$; SGA-A); mild or moderately malnourished ( $n=25$; SGA-B); and severely malnourished $(n=43$; SGA-C). Liver fibrosis markers, inflammatory indicator $\alpha$-Fetoprotein (AFP), tumor necrosis factor-alpha (TNF- $\alpha$ ), 25-hydroxyvitamin D, and PTH were estimated using immunoassay techniques. Results. CHC patients with moderate and severe malnutrition SGA scores showed a significant decline in the levels of vitamin D, increased PTH, and lower values of HGS and muscle mass indices compared to well-nourished patients and control subjects. In addition, malnutrition, vitamin D deficiency, and lower values of HGS, MAC, TSF, and MAMC showed significant correlation with liver severity among CHC patients. Liver fibrosis markers Hyp, HA, FN, APRI, HypI, HAI, and FNI as noninvasive biomarkers showed significant correlation with both severity of liver diseases and associated malnutrition, especially in cirrhotic HCV patients (F4) compared to those with significant fibrosis (F2-F3). Conclusion. The results showed that deficiency in vitamin D levels, HGS, SGA, and muscle mass scores (MAC, MAMC, or TSF) could be used as markers of liver pathogenicity in patients with CHC. In addition, the study concluded that noninvasive biomarkers Hyp, HA, FN, APRI, HypI, HAI, and FNI separately or in association with vitamin D status, HGS, SGA, and muscle mass scores (MAC, MAMC, or TSF) were significantly associated with an incidence of malnutrition between $\sim 70.5 \%$ and $89.6 \%$ of $\mathrm{CHC}$ patients with significant fibrosis and cirrhosis.
\end{abstract}

\section{Introduction}

In patients with hepatic viral infections (HCV and HBV), malnutrition was shown to be associated with morbidity and mortality particularly in patients with severe liver complications such as liver fibrosis, cirrhosis, cancer, and chronic viral hepatitis $(\mathrm{CHC})[1,2]$. In patients with compensated diseases and liver cirrhosis, malnutrition was significantly associated with liver diseases in $20 \%$ to $60 \%$ of the patients
[3]. In patients with $\mathrm{HCV}$, malnutrition was found early in association with viral infections and proceeds progressively throughout the spectrum of HCV disease [4].

In patients with $\mathrm{CHC}$, the rapid progression of liver damage was shown to be associated with malnutrition [5]. Frequently, $\mathrm{CHC}$ patients become malnourished since they are unable to meet their nutritional requirements [6].

In chronic liver disease, the pathogenesis of malnutrition is multifactorial and significantly includes a reduction in oral 
intake. Many consequences such as anorexia, nausea, dietary restrictions, altered nutrient biosynthesis, malabsorption, abnormalities of carbohydrate, lipid and protein metabolism, and a hypermetabolic state have significantly resulted from malnutrition [7].

In severe liver diseases, many effects of malnutrition like poor energetic intake due to a proinflammatory state and the presence of ascites along with lower levels of both calcium and magnesium were significantly provided in hepatic patients [3, 8]. Furthermore, nutrient digestion, absorption, storage, and metabolism were greatly affected in patients with chronic hepatic diseases, leading to deficiency in mineral and vitamin uptake and progressive protein-energy malnutrition [9]. Previous studies showed that deficiency in vitamin $\mathrm{D}$ levels along with disorder in the levels of parathyroid hormone $(\mathrm{PTH})$ was significantly associated with liver fibrosis and components of hepatic dysfunction in patients with $\mathrm{CHC}[10]$, whereas the natural history and the treatment of $\mathrm{CHC}$ infection were associated with the uptake and the levels of 25-vitamin D in the serum [11]. It was reported that intake of vitamin D alone or combined with interferonalpha significantly increases the innate and adaptive immune responses against $\mathrm{HCV}$ and suppresses $\mathrm{HCV}$ viral replication in Huh-7.5 cells [12]. The potential protective role of vitamin $\mathrm{D}$ proceeds via the expression of interferon-stimulated genes (ISGs) and a production of a synergistic effect with interferon-alpha.

Assessment of the degree of liver fibrosis correctly plays a pivotal role in controlling the progression of the disease and evaluating the prognosis for $\mathrm{CHC}$ infection as well as arranging for good new therapeutic strategies against $\mathrm{CHC}[13,14]$.

Histological examination was identified to be the gold standard methodology for diagnosis, assessing of the degree of hepatic fibrosis, and estimating prognosis among hepatic diseases [15]; however, in most circumstances, the use of liver biopsy in diagnosis sometimes results in false positive and false negative diagnoses among severe hepatic cases [16]. Thus, noninvasive methods were required for accurate diagnoses of liver fibrosis and cirrhosis as well as to differentiate between the early and past stages of liver fibrosis [17]. Noninvasive reliable biomarkers were shown to be an active area for clinical interest in the diagnosis of $\mathrm{CHC}$, grading hepatic fibrosis, and monitoring of outcome measures following the treatment of HCV-infected patients [18-20].

In recent studies, liver fibronectin, hydroxyproline, hyaluronic acid, liver function, and platelet counts and their respective indices like APRI, HA index, Hyp index, and FN index were significantly correlated with severity and liver fibrosis scores in different hepatic diseases particularly in CHC patients [18-21].

However, the correlation between these noninvasive biomarkers and their importance in predicting and evaluating malnutrition in $\mathrm{CHC}$ patients with severe hepatic dysfunction was not fully elucidated. Frequently, in patients with $\mathrm{CHC}$, the relation between malnutrition and severity of liver diseases is rarely undiagnosed $[4,21]$. In addition, there are no gold standard methods for the diagnosis of malnutrition in patients with $\mathrm{CHC}[22,23]$. Different parameters such as body mass index (BMI) and subjective global assessment
(SGA) along with the handgrip strength (HGS) were previously applied to evaluate malnutrition associated with liver cirrhosis [24-27].

In the current study, we aimed to elucidate the efficacy of serum liver fibrosis markers (fibronectin, hydroxyproline, and hyaluronic acid) and their respective indices (HA index, Hyp index, and FN index) and vitamin D status in predicting malnutrition associated with liver fibrosis in $\mathrm{CHC}$ patients and to investigate their association with the value of current clinical malnutrition assessment tools (SGA, BMI, MAMC, and HGS).

\section{Materials and Methods}

2.1. Subjects. A total of 80 patients aged 40-60 years with established chronic hepatitis $C$ virus for more than 6 years were recruited in this descriptive cross-sectional study. Patients with proven HCV antibodies, HCV-RNA positivity, and genotype determinations were included in this study.

In addition, age- and sex-matched 80 healthy subjects aged 40-60 years were selected as controls from a population undergoing a standard annual physical examination and biological measurements for medical insurance $[19,20]$. The data were collected from December 2012 to May 2013 from the outpatient department of Gastroenterology Surgical Centre, Faculty of Medicine, Mansoura University, Mansoura, Egypt. Patients with other liver complications such as HBV, HIV, cirrhosis status, hepatocellular carcinoma, alcohol intake, drug abuse, chronic renal insufficiency, chronic pancreatitis, and cognitive alterations like hepatic encephalopathy were excluded from this study. Also, patients who received medications that affect VitD3 metabolism were also excluded. Based on the ethical guidelines of the 1975 Declaration of Helsinki, the study protocol was reviewed and approved by ethical committee of Rehabilitation Research Chair (RRC), King Saud University, Kingdom of Saudi Arabia, under file number ID RRC-2016-092. An assigned informed consent was obtained from all participants prior to data collection. A heparinized syringe was used to collect blood samples from all subjects, and plasma samples were obtained from whole blood following centrifugation for $1 \mathrm{~min}$ at $1400 \mathrm{rpm}$. The samples were kept frozen at $20^{\circ} \mathrm{C}$ until use. Demographic and clinical data of the participants are in Table 1.

2.2. Anthropometric Measurements. A standardized procedures such as a tape measure and calibrated Salter Electronic Scales (Digital Pearson Scale; ADAM Equipment Inc., Columbia, MD, USA) were used to estimate both height and weight of all participants. BMI and waist to height ratio (WHtR) as parameters of adiposity were also calculated according previously validated universal cutoff values [28]. The Slim Guide skinfold caliper was used to measure the triceps skinfold, and the mean of three measurements was taken. Furthermore, the midarm circumference (MAC) was measured at the midpoint between the tip of the acromion and the olecranon process on the nondominant side of the body with a flexible tape measure with the subject sat upright with their arm flexed at $90^{\circ}[27,29]$. Midarm muscle 
TABLE 1: Demographic data, laboratory, physical activity, diet scores, and histological characteristics of chronic hepatitis C patients and healthy control subjects.

\begin{tabular}{|c|c|c|c|}
\hline Characteristics & All CHC patients, $N$, mean \pm SD (\%) & Healthy controls, $N$, mean \pm SD $(\%)$ & $p$ value \\
\hline No. & 80 & 80 & - \\
\hline Age (year) & $48.2 \pm 4.5$ & $47.8 \pm 4.5$ & 0.11 \\
\hline Gender (male/female) & $60 / 20$ & $60 / 20$ & 0.18 \\
\hline Anthropometry & & & 0.001 \\
\hline BMI & $21.8 \pm 3.2$ & $24.6 \pm 4.7$ & \\
\hline WHtR & $0.42 \pm 0.09$ & $0.75 \pm 0.13$ & \\
\hline Muscle mass & & & 0.001 \\
\hline MAC (in cm) & $23.3 \pm 2.6$ & $28.1 \pm 4.7$ & \\
\hline TSF (in mm) & $12.8 \pm 6.4$ & $16.3 \pm 3.1$ & \\
\hline MAMC (in mm) & $18.9 \pm 5.7$ & $22.9 \pm 4.2$ & \\
\hline Diet measurements & & & 0.001 \\
\hline Diet score & $11.3 \pm 3.8$ & $28.5 \pm 4.9$ & \\
\hline Dietary vitamin D intake (IU/d) & $86.8 \pm 48$ & $215 \pm 96$ & \\
\hline Dietary Ca intake (mg/d) & $650 \pm 89$ & $1400 \pm 185$ & \\
\hline SGA & & & 0.001 \\
\hline A (well nourished) & $12(15 \%)$ & $76(95 \%)$ & \\
\hline B (moderately malnourished) & $25(31.3 \%)$ & $4(5 \%)$ & \\
\hline C (severely malnourished) & $43(53.7 \%)$ & $0(0 \%)$ & \\
\hline PA & & & 0.001 \\
\hline Total PA (counts/min) & $398 \pm 75$ & $3850 \pm 480$ & \\
\hline MVPA (\%) & 14.6 & 85.9 & \\
\hline Total energy (kcal/d) & $1180 \pm 318$ & $6450 \pm 518$ & \\
\hline Sun exposure (h/day) & $1.4 \pm 0.6$ & $5.4 \pm 2.8$ & 0.001 \\
\hline Handgrip (kg) & & & 0.001 \\
\hline Right hand & $18.9 \pm 4.8$ & $41.9 \pm 4.8$ & \\
\hline Left hand & $22.6 \pm 3.7$ & $29.6 \pm 3.7$ & \\
\hline AST (IU/mL) & $68.4 \pm 24.7$ & $19.6 \pm 5.6$ & 0.001 \\
\hline $\operatorname{ALT}(\mathrm{IU} / \mathrm{mL})$ & $85.3 \pm 42.8$ & $23.5 \pm 4.3$ & 0.001 \\
\hline Albumin & $2.9 \pm 0.49$ & $4.5 \pm 1.8$ & 0.001 \\
\hline Bilirubin & $8.65 \pm 6.3$ & $0.85 \pm 0.29$ & 0.001 \\
\hline TNF- $\alpha(\mathrm{pg} / \mathrm{mL})$ & $34.7 \pm 6.8$ & $11.2 \pm 3.2$ & 0.001 \\
\hline AFP & $28.3 \pm 6.3$ & $4.6 \pm 1.9$ & 0.001 \\
\hline Vitamin 25(OH)D (ng/mL) & $14.7 \pm 3.2$ & $45.6 \pm 8.2$ & 0.001 \\
\hline PTH (pg/mL) & $56.2 \pm 4.1$ & $13.7 \pm 2.8$ & 0.001 \\
\hline Platelets $(109 / \mathrm{L})$ & $189 \pm 36.9$ & $315 \pm 51.7$ & 0.001 \\
\hline Duration of HCV (years) & $7.6 \pm 3.4$ & - & - \\
\hline HCV-RNA (IU/mL) & $12.8 \times 10^{5}$ & - & - \\
\hline HCV genotypes & & & - \\
\hline G4 & $62(77.5 \%)$ & - & \\
\hline G2 and 4 & $18(22 \%)$ & - & \\
\hline Viral load & $48.9 \pm 11.3$ & - & - \\
\hline Stage of fibrosis (METAVIR), $n(\%)$ & $74 / 80(92.3 \%)$ & - & - \\
\hline F0 & $6(7.5 \%)$ & & \\
\hline $\mathrm{F} 1$ & $12(15 \%)$ & & \\
\hline $\mathrm{F} 2$ & $15(18.75 \%)$ & & \\
\hline
\end{tabular}


TABLE 1: Continued.

\begin{tabular}{|c|c|c|c|}
\hline Characteristics & All CHC patients, $N$, mean \pm SD (\%) & Healthy controls, $N$, mean $\pm \mathrm{SD}(\%)$ & $p$ value \\
\hline F3 & $22(27.5 \%)$ & & \\
\hline $\mathrm{F} 4$ & $25(31.3 \%)$ & & \\
\hline Population, $n$ & & - & - \\
\hline F0-F1 & $18(22.5 \%)$ & & \\
\hline $\mathrm{F} 2-\mathrm{F} 4$ & $62(77.5 \%)$ & & \\
\hline F0-F3 & $55(68.75 \%)$ & & \\
\hline $\mathrm{F} 4$ & $25(31.3 \%)$ & & \\
\hline Mean length of liver biopsy core $(\mathrm{LBC}+\mathrm{SD})$ & $18.9 \pm 0.89 \mathrm{~cm}$ & - & - \\
\hline Mean number of portal tracts $(\mathrm{NoP}+\mathrm{SD})$ & $15 \pm 3.8$ & - & - \\
\hline Necroinflammation & & - & - \\
\hline $\mathrm{A} 0-\mathrm{A} 1$ & $25(31.3 \%)$ & & \\
\hline A2-A3 & $55(68.7 \%)$ & & \\
\hline
\end{tabular}

All values were reported as the mean \pm SD or median (interquartile range) or percentage. Kruskal-Wallis one-way ANOVA and post hoc (Tukey HSD) test were used to compare the mean values of the studied variables. Variables were considered significantly different at $P<0.05$. BMI: body mass index; WHtR: waist to height ratio; MVPA: moderate to vigorous physical activity; PA: physical activity; HGS: handgrip strength; SGA: subjective global assessment; AFP: $\alpha$-Fetoprotein; TNF- $\alpha$ : tumor necrosis factor-alpha; MAC: midarm circumference in cm; TSF: triceps skin fold thickness in mm; MAMC: midarm muscle circumference in $\mathrm{mm}$.

circumference (MAMC) was calculated using the MAC and the TSF according to standard equations given as follows [30]: $\{\mathrm{MAMC}=\operatorname{MAC}(\mathrm{cm})-(0.314 \times \operatorname{TSF}(\mathrm{mm}))\}[27,29]$.

2.3. Assessment of Liver Enzymes and HCV Markers. For all subjects, blood markers were performed on the day of biopsy or within 5 days after liver biopsy. Serum AST, ALT, total bilirubin, and albumin were performed using Max Discovery ${ }^{\mathrm{TM}}$ Color Endpoint Assay kits (Cat. No.BO-5605-01 and BO3460-08, Bioo Scientific Co., USA). $\alpha$-Fetoprotein (AFP) and tumor necrosis factor-alpha (TNF- $\alpha$ ) were estimated in the serum of all participants by using a sandwich ELISA assay and immune assay kits (AFP; R\&D Systems, USA; TNF- $\alpha$; BD Biosciences, USA, respectively). In addition, HCV antibody (anti-HCV) and HCV-RNA were estimated in patients with chronic HCV by using third-generation enzyme immunoassay (EIA) (Axsym HCV 3.0, Abbott Laboratories, Chicago, IL) and an in-house direct reverse transcriptasepolymerase chain reaction (RT-PCR) assay, respectively. Also, HCV genotypes were identified by a reverse hybridization method using Line Probe assay (INNO-LiPA HCV II kit, Innogenetics, Zwijndrecht, Belgium). The data were interpreted according to the manufacturer's instructions.

2.4. Assessment of Liver Fibrosis Markers. An enzyme-linked immunosorbent assay (ELISA) was performed to estimate both serum hyaluronic acid (HA) (HA-binding protein; Corgenix kit) for HA and hydroxyproline by using immune assay kits (Hyp; Cat. No. E0621Hu; Uscn Life Science Inc., Wuhan) for Hyp, respectively. Tissue fibronectin (FN) concentrations were estimated using an immune assay ELISA kit (ABIN1874233, Atlanta, GA30338, USA) at a wavelength of $450 \mathrm{~nm}$ using a spectrophotometer. The concentration of fibronectin was then determined by comparing the OD of the samples to the standard curve as previously reported $[19,20]$.
In addition, to predict liver fibrosis, different indices were calculated. ALT and AST indices were calculated by dividing the patient's test results by the upper limit of normal (40 IU/L) for the test. The AST/platelet count ratio index (APRI) was calculated as AST index/platelet count divided by $10^{3}$ times 100 . The HA, Hyp, and FN indices were calculated by dividing the patient's test results by platelet count ratio divided by $10^{3}$ times $100[17,19,31,32]$.

2.5. Assessment of Nutritional Scores. A prevalidated subjective global assessment (SGA) score with good to excellent interobserver reproducibility was used to calculate nutritional status in all subjects as previously reported [26, 33]. Based on the SGA score, CHC patients in this study were classified prospectively into three groups: well nourished $(n=12$; SGA-A), mild or moderately malnourished $(n=25$; SGA-B), and severely malnourished ( $n=43$; SGA-C) [33]. The control group reported a normal nutritional status (well nourished; SGA-A). Both SGA and body mass index (BMI) are used for the evaluation of malnutrition as previously reported [33].

2.6. Assessment of Handgrip Strength. Handgrip strength of both the right and left hands with $0.1 \mathrm{lbf}$ accuracy was measured by using a manual hydraulic dynamometer label JAMAR (Hydraulic Hand Dynamometer ${ }^{\circledR}$ Model PC-5030 J1, Fred Sammons, Inc., Burr Ridge, IL, USA) [34, 35]. The measurements were performed in the standard position, and each participant was seated in a straight-backed chair. Then, he was asked to squeeze the dynamometer two times with each hand. For each hand, approximately 2 min rest lapsed between trials and control for the effects of fatigue on each hand alternated, and the best value of two attempts was recorded [35]. The inter-rater technical error of measurement was less than $2.5 \%$ for both hands. Based on grip strength measurements (HGS), patients' muscle strength 
was expressed as normal (HGS: $\geq 300 \mathrm{mmHg}$ ), moderate (HGS: 231-299 mmHg), and low (HGS: 0-230 mmHg) [35].

2.7. Diet Information and Physical Activity. During the study period, all participants were instructed not to change their normal eating habits and record accurately the amount, type of food, and fluid consumed using food diaries. Then, dietary information for each participant were extensively referred according to reference dietary intakes for physically active people [36]. In addition, physical activity for each subject was evaluated during 7 consecutive days by using ACTi graph GT1M accelerometer (model WAM 7164; Fort Walton Beach, FL). The average intensity of PA was calculated from the total number of minutes each patient participated in sport activity different intensities. This intensity was based mainly on count thresholds and daily activity counts per minute. Subjects with less accelerometer counts $(\leq 100$ counts/min) were characterized by a sedentary lifestyle $[37,38]$.

According to energy expenditure, PA of all participants was classified into low or sedentary activity (thresholds are less than 4 metabolic equivalents (METs)), moderate activity (thresholds of 4 metabolic equivalents (METs)), and vigorous activity (thresholds of $7 \mathrm{METs}$ ), respectively, as previously mentioned, whereas 1 MET refers to either energy expenditure of $1 \mathrm{kcal} / \mathrm{kg} / \mathrm{h}$ or oxygen uptake in $3.5 \mathrm{~mL} / \mathrm{kg} / \mathrm{min}$ during a quiet sitting position [39].

2.8. Assessment of 25-Hydroxyvitamin D and PTH. From freshly separated serum samples of each patient, serum vitamin 25(OH)D level and intact PTH concentrations were estimated as previously reported $[25,26,28]$. A direct competitive chemiluminescence immunoassay with a Liaison auto-analyzer (Liaison, DiaSorin, Turin, Italy) was used to estimate both total 25-hydroxyvitamin (25(OH)D3) and intact PTH in serum of all participants. Based on the manufacturer's instruction, serum concentrations of $<10 \mathrm{ng} / \mathrm{mL}$ $25(\mathrm{OH}) \mathrm{D} 3$ were defined as severe VitD deficiency and $<30 \mathrm{ng} / \mathrm{mL} 25(\mathrm{OH}) \mathrm{D} 3$ as VitD insufficiency, whereas a range of $30-100 \mathrm{ng} / \mathrm{mL} 25(\mathrm{OH}) \mathrm{D} 3$ was considered normal.

2.9. Liver Histopathology. For all CHC patients to establish the diagnosis and the stage of liver injury, all patients underwent a percutaneous liver biopsy. An automatic 16-gauge tru-cut needle (biopsy gun) which provides adequate specimens for evaluation and fewer cases with tissue fragmentations was used for taking liver biopsies as previously mentioned [16, 40]. For better histological evaluation, the analyzed specimens of liver biopsy should at least 15$25 \mathrm{~mm}$ long with complete portal tracts (10 CPTs) [40]. Formalin-fixed, paraffin-embedded sections were stained with hematoxylin and eosin and with Masson's Trichrome. Slides were labeled with patient identification numbers and then reviewed and graded blindly by a senior pathologist; the mean length of liver biopsy and the number of portal tracts were assessed (including only the complete, intact portal tracts) [40]. The degree of fibrosis was scored according to the METAVIR system, and no fibrosis was defined as F0, mild fibrosis as F1, moderate fibrosis as F2, severe fibrosis as F3, and cirrhosis as F4. Significant fibrosis was also defined as F2-F4. Hepatic inflammatory activity was also scored [40].

2.10. Statistical Analysis. The statistical power calculations for the selected sample size of 160 subjects were shown to identify a power of $98 \%$ and a significance level of 0.05 with an expected frequency of $8.5 \%$.

The results obtained were expressed as the mean and standard deviation. Among groups, Kruskal-Wallis oneway ANOVA and post hoc (Tukey HSD) test were used to compare the mean values of the studied variables. Additionally, Spearman's rank correlation analysis was performed to assess the relationship between various study parameters. The predictive values of vitamin D status; liver fibrosis markers (fibronectin (FN), hydroxyproline (Hyp), and hyaluronic acid (HA)) and their respective indices (HA index, Hyp index, and FN index); and the current clinical malnutrition assessment tools (SGA, BMI, MAMC, and HGS) were examined using stepwise linear regression analysis. Variables that have the highest $R$-squared and strong significance were added in this model. Only, in this study, FN, HA, HAI, HypI, FNI, APRI, vitamin D, Ca and vitamin D intake, SGA, BMI, MAMC, and HGS showed higher $R$-squared and strong significance, whereas PA, basal metabolic rate (BMR kcal/day), total energy expenditure (TEE, kcal/day), and sun exposure showed lower $R$-squared and were deleted from the proposed model. A statistical software SPSS version 18 was used, and the data obtained were deemed significant at $p<0.05$.

\section{Results}

A total of $80 \mathrm{CHC}$ patients were included in this study (Table 1). The mean age of the patients was $48.2 \pm 4.5$, and most of them are male (75\%). Based on molecular and genotype analysis, the most frequently detected genotype was 4 (77.5\%) along with $22.5 \%$ of patients with mixed HCV genotypes 2 and 4 . Positive HCV patients showed HCV overloads of $48.9 \pm 11.3$ with higher expression of HCV-RNA (IU/mL) $\left(12.8 \times 10^{5}\right)$. The significant fibrosis was found in $92.3 \%$ of $\mathrm{CHC}$ patients using the METAVIR system. Most of patients had grade A2-A3 inflammation $(68.7 \%)$, while only a minority had grade A0-A1 with $31.3 \%$.

On the other hand, liver biopsies from the majority of patients had stage F0-F1 (22.5\%) of mild fibrosis and stage (F2-4) (77.5\%) of significant fibrosis, while only about $31.3 \%$ had cirrhosis (F4) (Table 1). Also, patients with $\mathrm{CHC}$ virus showed lower values of adiposity markers BMI and WHtR compared to healthy controls.

Laboratory confirming biomarkers were also estimated in $\mathrm{CHC}$ patients and control subjects. $\mathrm{CHC}$ patients showed a significant increase in the levels of AST, ALT, TNF- $\alpha$, AFP, and bilirubin and a decline in the values of albumin and platelet counts compared to healthy control subjects as shown in Table 1. Similarly, a decrease in serum vitamin 25(OH)D (ng/mL) levels and an increase in PTH $(\mathrm{pg} / \mathrm{mL})$ levels were significantly reported in CHC patients compared to control subjects.

In addition, using SGA scores, malnutrition was estimated among CHC patients (Table 1). Only $15 \%$ of the 
patients showed normal nutrition (SGA score A), and $85 \%$ of the study population had moderate to severe SGA scores (B and C) of malnutrition; they are classified into $31.3 \%$ with moderately malnourished SGA score (B) and 53.7\% with severe malnourished SGA score (C). In CHC patients, malnutrition was shown to be significantly $(p=0.001)$ linked with poor PA levels, daily sunexposure, diet scores, and lower adminstration of both vitamin D and Ca intakes.

In this study, muscle mass and strength were estimated as measures of functional capacity associated with malnutrition in CHC patients (Table 1). Thus, HGS of both arms and MAC, TSF, and MAMC were measured in all CHC patients and control subjects. Compared to control subjects, lower significant values of HGS, MAC, TSF, and MAMC were reported in $\mathrm{CHC}$ patients. The data confirmed significant functional loss in muscle mass and physical performance in CHC patients compared to control subjects (Table 1). This may be attributed to malnutrition, lower vitamin $\mathrm{D}$, and $\mathrm{Ca}$ intake which leads to reduction in body cell mass.

Based on assessed malnutrition scores (SGA scores), a significant increase in the levels of AST, ALT, TNF- $\alpha$, AFP, bilirubin, and INR and a decline in the values of BMI, WHtR, albumin, and platelet counts were reported in $\mathrm{CHC}$ patients with moderate (B) and severe (C) SGA scores compared to those who had normal diets (Table 2).

Also, in moderate and severely malnourished $\mathrm{CHC}$ patients who received diets containing insufficient vitamin $\mathrm{D}$ and $\mathrm{Ca}$, a significant increase in vitamin $25(\mathrm{OH}) \mathrm{D}$ $(\mathrm{ng} / \mathrm{mL})$ levels and an increase in the levels of PTH $(\mathrm{pg} / \mathrm{mL})$ were significantly reported compared to control subjects and $\mathrm{CHC}$ patients who had normal diets with adequate vitamin $\mathrm{D}$ and $\mathrm{Ca}$ as shown in Table 2 and Figure 1(a). In the same line, vitamin $\mathrm{D}$ deficiency was shown to be significantly increased in malnourished $\mathrm{CHC}$ patients who received diets with inadequate vitamin $\mathrm{D}$ supplements compared to control and $\mathrm{CHC}$ with normal diets as in Figures 1(b) and 1(c). Similarly, poor PA levels with lower TE were significantly correlated with inadequate diets and vitamin $\mathrm{D}$ in moderate and severely malnourished CHC patients, whereas PA scores, TE (kcal/d), and MVPA (\%) were significantly reduced in CHC patients with A $(p=0.01), \mathrm{B} \quad(p=0.01)$, and $\mathrm{C}$ $(p=0.001)$ nutritional SGA scores compared to healthy controls (Figure 1(d)).

Also, HGS scores of both arms, MAC, TSF, and MAMC as outcome measures of functional loss in muscle mass and physical performance were reported to be closely correlated with malnutrition SGA scores in CHC patients. HGS scores were significantly reduced in $\mathrm{CHC}$ patients with severe $(p=0.001)$ and moderate $(p=0.01)$ malnutrition compared to those of normal nutrition $(p=0.01)$ and control groups, respectively (Figure $2(\mathrm{a})$ ). In addition, muscle mass indices (MAC, $p=0.05$; TSF, $p=0.01$; and MAMC, $p=0.001$ ) showed significant reduction in moderate and severely malnourished $\mathrm{CHC}$ patients compared to those who had normal diets and control subjects (Figure 2(b)).

In this current study, HYP, HA, and $\mathrm{FN}$ as markers of liver fibrosis were estimated in CHC patients. HYP, HA, and $\mathrm{FN}$ were significantly increased in $\mathrm{CHC}$ patients with A $(p=0.01), \mathrm{B}(p=0.01)$, and C $(p=0.001)$ nutritional
SGA scores compared to healthy controls (Figure 2(c)). Also, expression of HCV-RNA (IU/mL) and viral overloads significantly increased in moderate and severely malnourished $\mathrm{CHC}$ patients compared to those who were well nourished as shown in Figure 2(d).

Regarding the presence of significant fibrosis and cirrhosis, CHC patients with significant cirrhosis (F4) showed significant $(p=0.001)$ increase in the levels of AST, ALT, TNF- $\alpha$, AFP, bilirubin, INR, APRI, Hyp index, HA index, and FN index; a decline in the values of vitamin $25(\mathrm{OH}) \mathrm{D}$ $(\mathrm{ng} / \mathrm{mL})$; and an increase in the levels of PTH $(\mathrm{pg} / \mathrm{mL})$ with reduction in platelet counts compared to $\mathrm{CHC}$ patients with mild (F0-F1) and significant fibrosis (F2-F4), respectively (Table 3).

Also, higher functional loss in muscle mass and physical performance was reported in $\mathrm{CHC}$ patients with significant cirrhosis (F4). HGS scores of both arms, MAC, TSF, and MAMC were significantly $(p=0.001)$ reduced with lower vitamin $\mathrm{D}$ and Ca intake in malnourished $\mathrm{CHC}$ patients with significant cirrhosis (F4) compared to $\mathrm{CHC}$ patients with mild (F0-F1) and significant (F2-F4) fibrosis, respectively (Table 3).

Malnutrition, adiposity, handgrip strength, muscle mass, and vitamin $\mathrm{D}$ and $\mathrm{Ca}$ intake were shown to be significantly correlated with the degree of liver fibrosis in $\mathrm{CHC}$ patients. The degree of significant fibrosis and cirrhosis correlated positively with adiposity, lower vitamin $25(\mathrm{OH}) \mathrm{D}$, and increased levels of PTH, diet scores, and vitamin $\mathrm{D}$ and $\mathrm{Ca}$ intake and negatively with SGA malnutrition scores, HGS, and muscle mass indices (MAC, TSF, and MAMC, respectively) (Table 4).

The data also, confirmed that Hyp, HA, FN, APRI, HypI, $\mathrm{HAI}$, and FNI as noninvasive biomarkers could be used separately or in association with vitamin D status and HGS as predictors for both liver fibrosis and malnutrition in $\mathrm{CHC}$ patients. Stepwise regression analysis revealed that adiposity, liver fibrosis markers (Hyp, HA, FN, APRI, HypI, HAI, and FNI), muscle mass scores (MAC, MAMC, TSF, HGS, and SGA), and vitamin D deficiency were associated with $\sim 70.5 \%-89.6 \%$ of the incidence of malnutrition in $\mathrm{CHC}$ patients with significant fibrosis and cirrhosis (Table 5).

\section{Discussion}

In this study, malnutrition was cross-sectionally surveyed in $80 \mathrm{CHC}$ patients and was shown to be significantly associated with severity of liver fibrosis. Patients with well-nourished status was predicted in $15 \%$ of the study population, and $85 \%$ of the study population had major changes in nutritional status; they were classified into $31.3 \%$ with a moderate malnourished SGA score (B), and $53.7 \%$ of the study population had a severe malnourished SGA score (C). In addition, the results revealed that adiposity, liver fibrosis markers (Hyp, HA, FN, APRI, HypI, HAI, and FNI), muscle mass scores (MAC, MAMC, TSF, HGS, and SGA), and vitamin $\mathrm{D}$ deficiency were associated significantly with $\sim 70.5 \%-$ $89.6 \%$ of the incidence of malnutrition in CHC patients with significant fibrosis and cirrhosis. 
TABLE 2: Comparison of adiposity, diets, and other laboratory biomarkers in CHC patients based on nutritional assessment scores (SGA scores).

\begin{tabular}{|c|c|c|c|c|}
\hline \multirow{2}{*}{ Variables } & \multicolumn{3}{|c|}{ SGA classes $($ mean $\pm \mathrm{SD})$} & \multirow{2}{*}{$p$ value } \\
\hline & $\mathrm{A}(n=12)$ & $\mathrm{B}(n=25)$ & $\mathrm{C}(n=43)$ & \\
\hline Anthropometry & & & & 0.001 \\
\hline BMI & $21.2 \pm 3.9$ & $18.1 \pm 2.8$ & $16.9 \pm 2.5$ & \\
\hline WHtR & $0.69 \pm 0.12$ & $0.49 \pm 0.84$ & $0.29 \pm 0.68$ & \\
\hline Diet measurements & & & & 0.001 \\
\hline Diet score & $10.9 \pm 3.6$ & $8.2 \pm 3.2$ & $6.3 \pm 2.1$ & \\
\hline Dietary vitamin D intake (IU/d) & $89.5 \pm 51$ & $72.8 \pm 48$ & $65.8 \pm 32$ & \\
\hline Dietary Ca intake (mg/d) & $696 \pm 94$ & $636 \pm 41$ & $548 \pm 26$ & \\
\hline AST (IU/mL) & $62.3 \pm 21.5$ & $68.1 \pm 21.9$ & $76.3 \pm 23.4$ & 0.001 \\
\hline $\operatorname{ALT}(\mathrm{IU} / \mathrm{mL})$ & $79.8 \pm 31.2$ & $82.4 \pm 28.3$ & $92.7 \pm 28.7$ & 0.001 \\
\hline Albumin & $3.7 \pm 0.6$ & $2.97 \pm 0.49$ & $2.48 \pm 0.31$ & 0.001 \\
\hline Bilirubin & $2.86 \pm 5.9$ & $5.8 \pm 2.6$ & $8.96 \pm 6.3$ & 0.001 \\
\hline TNF- $\alpha(\mathrm{pg} / \mathrm{mL})$ & $33.9 \pm 4.8$ & $38.2 \pm 5.1$ & $48.7 \pm 6.3$ & 0.001 \\
\hline AFP & $29.8 \pm 5.3$ & $32.2 \pm 3.7$ & $41.9 \pm 6.8$ & 0.001 \\
\hline INR & $1.25 \pm 0.18$ & $1.8 \pm 1.1$ & $2.5 \pm 1.18$ & 0.001 \\
\hline Platelets (109/L) & $192 \pm 32.7$ & $178 \pm 18.3$ & $96 \pm 16.7$ & 0.001 \\
\hline
\end{tabular}

All values were reported as the mean \pm SD or median (interquartile range) or percentage. Kruskal-Wallis one-way ANOVA and post hoc (Tukey HSD) test were used to compare the mean values of the studied variables. Variables were considered significantly different at $P<0.05$. BMI: body mass index; WHtR: waist to height ratio; SGA: subjective global assessment; AFP: $\alpha$-Fetoprotein; TNF- $\alpha$ : tumor necrosis factor-alpha; INR: international normalized ratio of prothrombin.

Malnutrition shows variability from $20 \%$ in patients with compensated disease to more than $60 \%$ in patients with liver cirrhosis, which depends mainly on the type of the method used for evaluation [2-4]. In patients with chronic hepatitis $\mathrm{C}(\mathrm{CHC})$, malnutrition was reported early in the course of $\mathrm{HCV}$ and proceeds progressively throughout the spectrum of HCV disease [4].

In $\mathrm{CHC}$ patients, the rapid progression of liver damage was shown to be associated with malnutrition [5]. Frequently, $\mathrm{CHC}$ patients become malnourished whereas they are unable to meet their nutritional requirements [6].

$85 \%$ of our patients were malnourished (SGA B or C) which was higher than the reported $28 \%$ percentage previously estimated in cirrhotic patients [41]. The difference may be partially explained on the basis of the difference in clinical severity of liver fibrosis among our CHC patients. Similar to our SGA prevalence rates, other reported studies showed statistically significant differences in the BMI and overall score of SGA throughout the three times of SGA assessment. According to these studies, chronic hepatitis $\mathrm{C}$ study subjects lying in category A were $83.3 \%, 63.7 \%$, and $75.5 \%$ in the 1st, 2nd, and 3rd SGA assessment trials, respectively, and the rest of $\mathrm{CHC}$ patients fall in category B. Their data showed that nutritional assessment is authoritative for better clinical outcome in patients with chronic hepatitis C treated with combination therapy [42].

In addition, in this study, significant correlation was reported between estimated SGA scores and severity of liver fibrosis as measured by increased liver dysfunction, AST, ALT, TNF- $\alpha$, AFP, bilirubin, INR; a decline in the values of BMI, WHtR, and albumin; and a significant increase in the levels of Hyp, HA, and FN and their respective indices (APRI, Hyp, HAI, and FNI) in CHC patients with significant fibrosis and cirrhosis. In previous studies, a similar correlation was significantly $(p=005)$ reported between SGA and prognosis of cirrhosis as measured by Child-Pugh scores [30]. Recently, it was reported that malnutrition was significantly associated with severity of liver fibrosis and that nutritional SGA scores could be used to predict liver severity and short-term survival in cirrhotic patients [24].

Vitamin D is the best well-known naturally regulators for both bone mineralization and calcium homeostasis [43]. In addition, it has many other pivotal physiological roles in living cells, such as cellular proliferation, apoptosis, differentiation, and inflammation [43].

In patients with $\mathrm{HCV}$ infection, vitamin $\mathrm{D}$ status was shown to be inversely correlated with liver fibrosis progression, liver dysfunction, and cellular damage in treated and nontreated naive $\mathrm{CHC}$ stages [44]. Lower serum vitamin D levels have been reported in patients with chronic liver disease from different etiologies [45]. The deficiency in vitamin $\mathrm{D}$ levels was associated with the pathogenesis of liver cells in $46 \%$ to $92 \%$ of CHC patients [45].

In this study, a reduction in serum level of vitamin 25(OH)D and an increase in PTH levels were significantly reported in CHC patients compared to control subjects. Deficiency of vitamin 25(OH)D and increased levels of PTH were significantly correlated to liver severity. Significant reduction in the levels of vitamin $25(\mathrm{OH}) \mathrm{D}$ and increased levels of PTH 


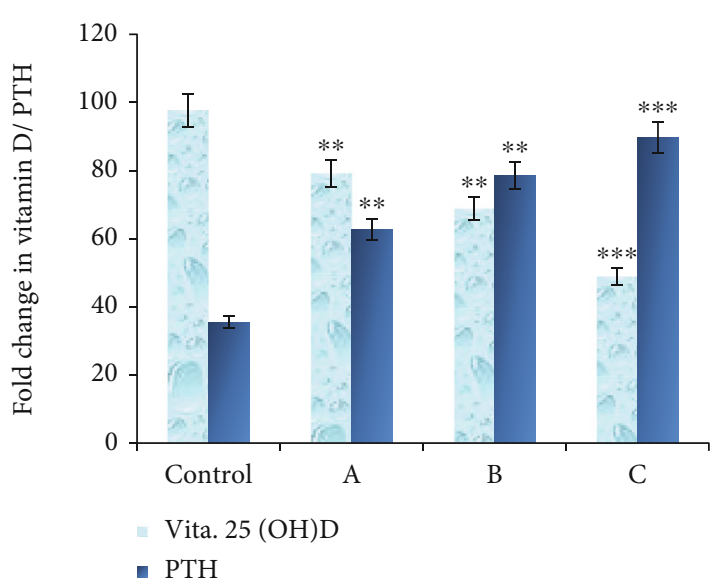

(a)

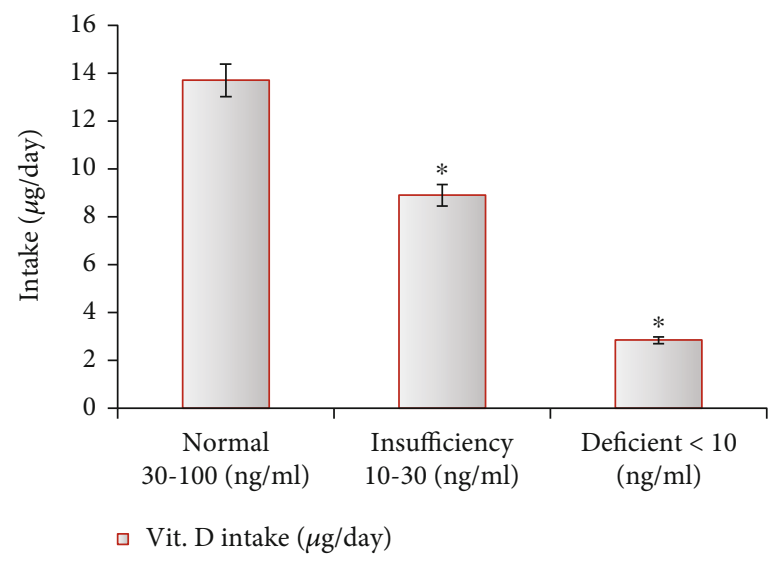

(c)

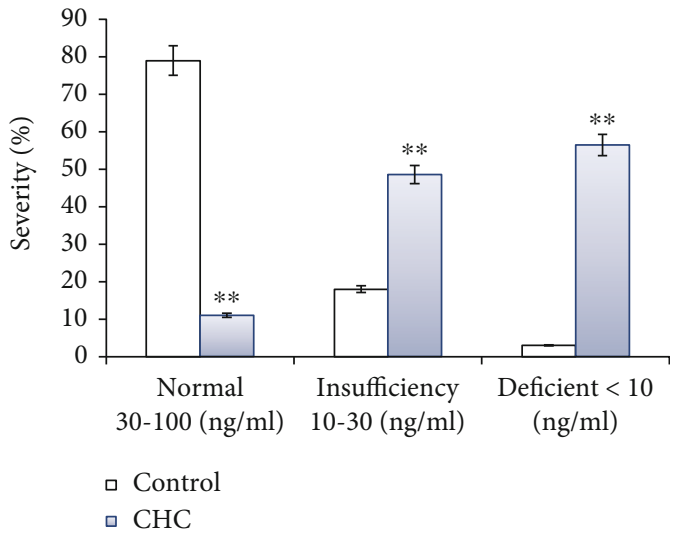

(b)

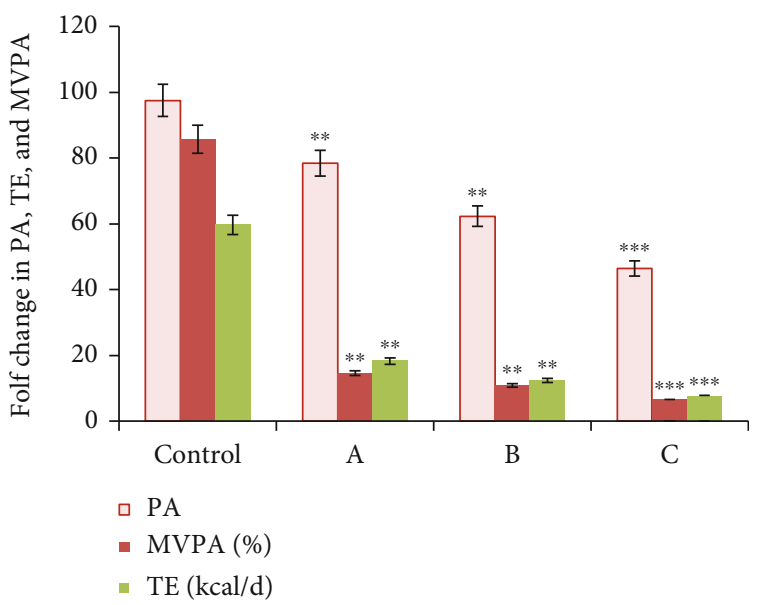

(d)

FIGURE 1: Serum vitamin 25(OH)D and PTH levels, vitamin D deficiency score, related vitamin D intake, and physical activity (PA) scores for healthy controls and CHC patients with different nutritional assessment scores; SGA scores (a-c). (a) Significant difference in serum vitamin 25(OH)D and PTH levels in CHC patients with A $(p=0.01), \mathrm{B}(p=0.01)$, and C $(p=0.001)$ nutritional SGA scores compared to healthy controls. (b) Severity of vitamin $25(\mathrm{OH})$ deficiency among control and CHC patients. CHC patients showed higher ratios of vitamin $25(\mathrm{OH}) \mathrm{D}$ insufficiency and deficiency $(p=0.01)$ compared to control subjects. $(c)$ Lower vitamin D intake was significantly reported in $\mathrm{CHC}$ patients with both insufficiency and deficiency in vitamin $25(\mathrm{OH}) \mathrm{D}$ levels $(p=0.05)$. (d) PA scores, TE (kcal/d), and MVPA (\%) were significantly reduced in CHC patients with A $(p=0.01), \mathrm{B}(p=0.01)$, and C $(p=0.001)$ nutritional SGA scores compared to healthy controls. PTH: parathyroid hormone; MVPA: moderate to vigorous physical activity; PA: physical activity; SGA: subjective global assessment; A: well nourished; B: moderately malnourished; C: severely malnourished; TE: total energy (kcal/d).

were significantly reported in cirrhotic $\mathrm{CHC}$ cases compared to those with significant fibrosis.

Previously, it was reported that adequate vitamin D levels obtained through normal diets or by supplements significantly affect HCV replication in liver cells via immunomodulatory effect $[10,11,44,45]$. Also, vitamin $\mathrm{D}$ had a direct inhibitory effect on viral production by regulating the expression of interferon-beta (INF- $\beta$ ) and consequent increase of innate immune response of infected liver cell against further $\mathrm{HCV}$ propagation. Based on this study, deficiency in vitamin $\mathrm{D}$ among our CHC patients results in a reduction in immunity which gives a good universe for more expression and prognosis of $\mathrm{HCV}$, resulting in severe liver fibrosis $[44,45]$.

In addition, in our $\mathrm{CHC}$ patients with cirrhosis, an increase in the levels of AFP and TNF- $\alpha$ as inflammatory cytokines was recorded; this may be due to the fact that vita- min $\mathrm{D}$ deficiency produces an increase in intrahepatic inflammation, circulating levels of several inflammatory cytokines, and chemokines closely related to disease progression as previously reported [44-46].

Biological activity of vitamin D in normal cells were performed in combination with several other hormones, including parathyroid hormone (PTH). During vitamin D deficiency, levels of ionized calcium were declined [47]. This in turn activates the increase of PTH levels to compensate loss of calcium via stimulation of bone resorption to the release of calcium. Thus, PTH level is often used as an index of vitamin D repletion whereas adequacy of vitamin D levels is obtained in normal cells when PTH levels are maximally suppressed [46-49].

Previously, vitamin D deficiency in $\mathrm{HCV}$-infected patients was inversely correlated with elevated PTH serum 


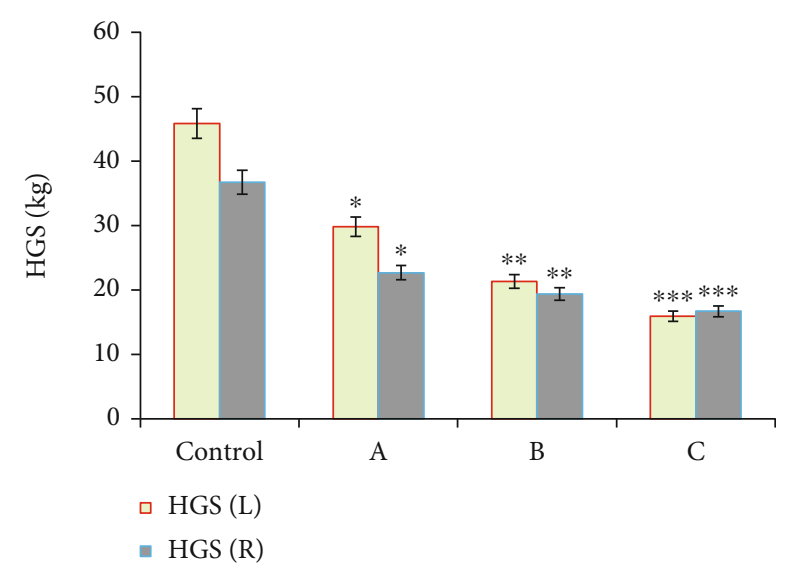

(a)

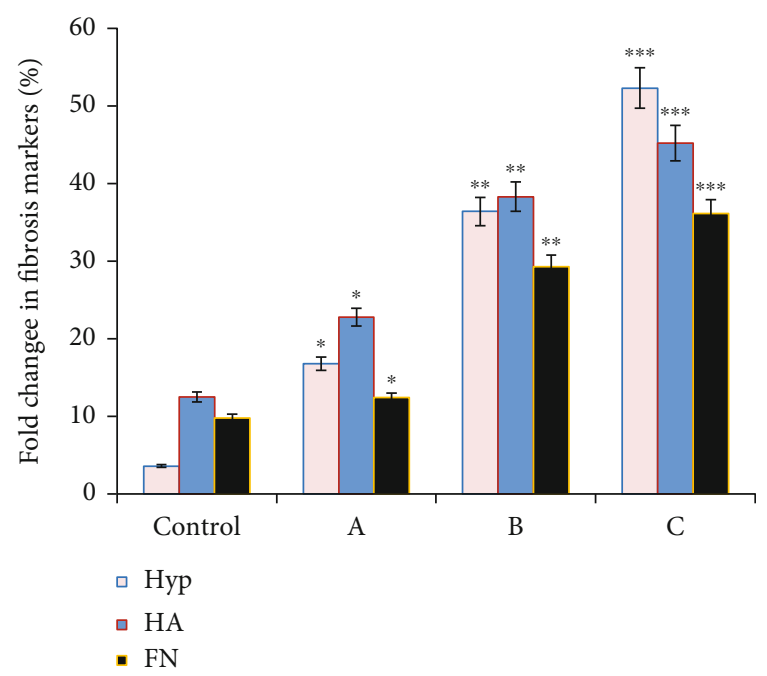

(c)

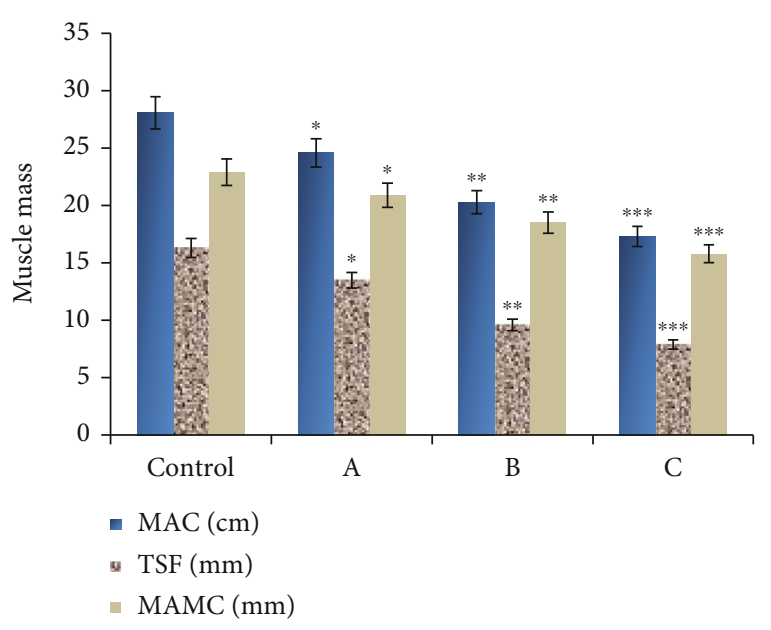

(b)

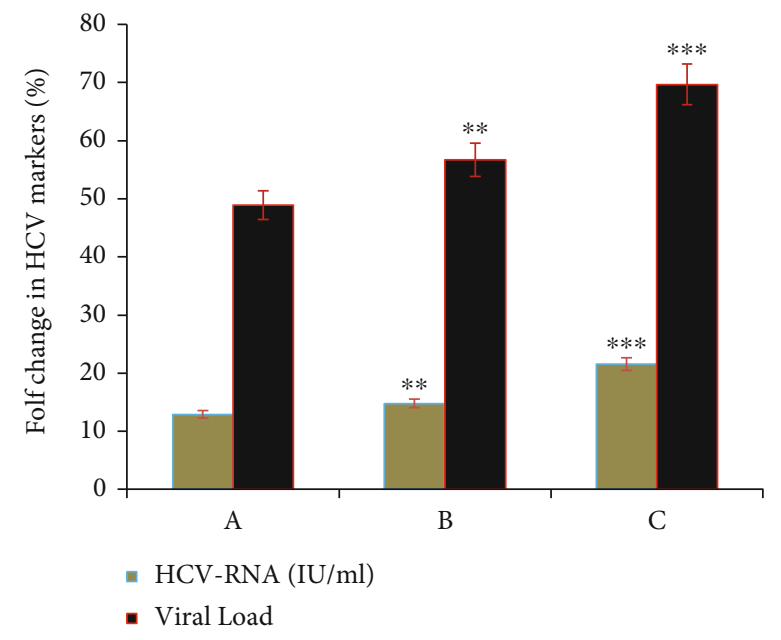

(d)

FIGURE 2: Handgrip strength, muscle mass parameters (MAC, TSF, and MAMC), and related HCV markers and liver fibrosis markers in healthy control and CHC patients based on different nutritional assessment scores; SGA scores (A: well nourished; B: moderately malnourished; C: severely malnourished). (a) HGS scores were significantly reduced in CHC patients with severe $(p=0.001)$ and moderate $(p=0.01)$ malnutrition compared to those of the normal nutrition $(p=0.01)$ and control groups, respectively. (b) Muscle mass indices (MAC, $p=0.05$; TSF, $p=0.01$; and MAMC; $p=0.001$ ) showed significant reduction in all CHC patients with normal and malnutrition SGA scores compared to control subjects with normal nutrition scores. (c) The levels of HYP, HA, and FN as markers of liver fibrosis were significantly increased in CHC patients with A $(p=0.01), \mathrm{B}(p=0.01)$, and C $(p=0.001)$ nutritional SGA scores compared to healthy controls. (d) HCV-RNA (IU/mL) and HCV viral load as related HCV markers were significantly highly expressed in CHC patients with moderate (SAG B score; $p=0.01$ ) and severe (SAG C score; $p=0.001$ ) malnutrition compared to those who were well nourished (SAG A score). MAC: midarm circumference in $\mathrm{cm}$; TSF: triceps skin fold thickness in mm; MAMC: midarm muscle circumference in mm; CHC patients: chronic hepatitis C patients; HYP: hydroxyproline; HA: hyaluronic acid; FN: fibronectin; SGA: subjective global assessment; A: well nourished; B: moderately malnourished; C: severely malnourished.

levels. In normal control subjects with vitamin $\mathrm{D}$ values above approximately $30 \mathrm{ng} / \mathrm{mL}$, serum PTH levels were shown to be at low steady levels [1-11].

The changes in the levels of vitamin $25(\mathrm{OH}) \mathrm{D}$ and PTH levels were significantly correlated with malnutrition in $\mathrm{CHC}$ patients. In this study, $\mathrm{CHC}$ patients with moderate and severe malnutrition ASG score showed a significant decline in vitamin D and an increase in PTH levels, especially in $\mathrm{CHC}$ patients who received inadequate amounts of vitamin $\mathrm{D}$ and $\mathrm{Ca}$ in their foods.
The data obtained were in line with those who reported that the incidence of liver diseases significantly influences physiological process relating to nutrient digestion, absorption, storage, and metabolism. These collectively may lead to deficiency in vitamin, mineral, and protein-energy malnutrition $[2,4,5$, 12]. Previous research studies showed that hepatitis $C$ patients with or without liver complications are already at risk of malnutrition and inadequate dietary intake and that nutrient deficiencies especially those of vitamin D and Ca have a direct impact on the clinical history of patients with liver disease $[7,33]$. 
TABLE 3: Association of malnutrition, vitamin D deficiency, handgrip strength, muscle mass, and other related liver fibrosis markers with the presence of significant fibrosis and cirrhosis in CHC.

\begin{tabular}{|c|c|c|c|c|c|c|}
\hline \multirow{2}{*}{ Variables } & \multicolumn{2}{|c|}{ Significant fibrosis $($ mean \pm SD) } & \multirow{2}{*}{$p$ value } & \multicolumn{2}{|c|}{ Cirrhosis $($ mean $\pm \mathrm{SD})$} & \multirow{2}{*}{$p$ value } \\
\hline & $\mathrm{F} 0-\mathrm{F} 1$ & F2-F4 & & F0-F3 & $\mathrm{F} 4$ & \\
\hline AST (IU/mL) & $65.7 \pm 11.3$ & $81.7 \pm 16.7$ & 0.001 & $78.3 \pm 12.4$ & $125.3 \pm 18.4$ & 0.001 \\
\hline $\mathrm{ALT}(\mathrm{IU} / \mathrm{mL})$ & $72.7 \pm 6.8$ & $112 \pm 11.8$ & 0.001 & $100 \pm 9.8$ & $186 \pm 21.3$ & 0.001 \\
\hline INR & $1.2 \pm 0.14$ & $1.3 \pm 1.1$ & 0.001 & $2.6 \pm 0.61$ & $3.7 \pm 1.8$ & 0.001 \\
\hline Platelets (109/L) & $228.6 \pm 21.3$ & $210.8 \pm 14.2$ & 0.001 & $198.6 \pm 11.7$ & $161.2 \pm 31.8$ & 0.001 \\
\hline HYP $(\mathrm{lg} / \mathrm{mL})$ & $2.9 \pm 1.9$ & $8.6 \pm 3.65$ & 0.001 & $15.2 \pm 4.9$ & $18.2 \pm 3.9$ & 0.001 \\
\hline HA (ng/mL) & $49.5 \pm 10.2$ & $136.0 \pm 31.2$ & 0.001 & $186.5 \pm 38.4$ & $215.7 \pm 48.7$ & 0.001 \\
\hline $\mathrm{FN}(\mathrm{ng} / \mathrm{mL})$ & $16.5 \pm 3.6$ & $31.5 \pm 3.6$ & 0.001 & $61.8 \pm 12.8$ & $86.9 \pm 21.3$ & 0.001 \\
\hline APRI & $0.8 \pm 0.45$ & $2.6 \pm 0.9$ & 0.001 & $2.85 \pm 086$ & $3.6 \pm 0.51$ & 0.001 \\
\hline Hyp index & $1.96 \pm 0.42$ & $5.81 \pm 1.2$ & 0.001 & $8.96 \pm 1.6$ & $12.9 \pm 3.31$ & 0.001 \\
\hline FNPRI index & $2.8 \pm 0.68$ & $7.9 \pm 2.7$ & 0.001 & $9.6 \pm 1.6$ & $14.9 \pm 6.1$ & 0.001 \\
\hline HAPRI & $0.86 \pm 0.56$ & $1.68 \pm 1.3$ & 0.001 & $1.8 \pm 0.86$ & $4.9 \pm 1.69$ & 0.001 \\
\hline Vitamin $25(\mathrm{OH}) \mathrm{D}(\mathrm{ng} / \mathrm{mL})$ & $21.7 \pm 2.9$ & $12.3 \pm 3.9$ & 0.001 & $15.2 \pm 1.5$ & $9.8 \pm 2.3$ & 0.001 \\
\hline PTH $(\mathrm{pg} / \mathrm{mL})$ & $48.5 \pm 3.1$ & $62.2 \pm 6.3$ & 0.001 & $86.7 \pm 2.6$ & $96.7 \pm 1.9$ & 0.001 \\
\hline Dietary vitamin D intake (IU/d) & $96.4 \pm 48$ & $81.9 \pm 31$ & 0.001 & $56.8 \pm 31$ & $46.7 \pm 25$ & 0.001 \\
\hline Dietary Ca intake (mg/d) & $615 \pm 68$ & $549 \pm 74.5$ & 0.001 & $510 \pm 31.6$ & $396 \pm 36.1$ & 0.001 \\
\hline Diet score & $12.8 \pm 1.9$ & $10.3 \pm 2.8$ & 0.001 & $9.6 \pm 3.4$ & $6.1 \pm 4.7$ & 0.001 \\
\hline \multicolumn{7}{|l|}{ SGA } \\
\hline A (well nourished) & 6 & 12 & 0.001 & 15 & 0 & 0.001 \\
\hline B (moderately malnourished) & 7 & 23 & & 16 & 11 & \\
\hline C (severely malnourished) & 5 & 27 & & 24 & 14 & \\
\hline \multicolumn{7}{|l|}{ Handgrip (kg) } \\
\hline Right hand & $21.6 \pm 5.3$ & $18.1 \pm 3.6$ & 0.001 & $17.8 \pm 3.7$ & $16.8 \pm 1.6$ & 0.001 \\
\hline Left hand & $23.1 \pm 3.7$ & $16.5 \pm 2.1$ & & $15.8 \pm 2.4$ & $14.2 \pm 3.8$ & \\
\hline \multicolumn{7}{|l|}{ Muscle mass scores } \\
\hline MAC (in cm) & $21.5 \pm 1.8$ & $19.7 \pm 3.6$ & 0.001 & $17.9 \pm 3.1$ & $16.9 \pm 1.6$ & 0.001 \\
\hline TSF (in mm) & $13.6 \pm 5.2$ & $12.1 \pm 3.8$ & & $11.6 \pm 3.8$ & $9.8 \pm 4.6$ & \\
\hline MAMC (in mm) & $17.6 \pm 3.7$ & $16.9 \pm 2.3$ & & $15.9 \pm 2.8$ & $14.9 \pm 6.3$ & \\
\hline
\end{tabular}

SD: standard deviation; APRI: AST to platelet ratio index; Hyp index: hydroxyproline to platelet ratio index; HAPRI: HA to platelet ratio index; FNPRI: FN to platelet ratio index; ALT: alanine aminotransferase; AST: aspartate aminotransferase; PTH: parathyroid hormone; MAC: midarm circumference in cm; TSF: triceps skin fold thickness in mm; MAMC: midarm muscle circumference in mm; CHC patients: chronic hepatitis C patients; HYP: hydroxyproline; HA: hyaluronic acid; FN: fibronectin; SGA: subjective global assessment; A: well nourished; B: moderately malnourished; C: severely malnourished; INR: international normalized ratio of prothrombin. Student's $t$-test was used followed by Mann-Whitney $U$ test. $P$ values at $<0.05$ are considered statistically significant.

Thus, diagnosing and treating malnutrition associated with $\mathrm{HCV}$ caused before the onset of complications particularly cirrhosis and hepatocellular carcinoma are significantly needed for new methods for assessing nutritional status [7, 29, 33].

In this study, HGS scores of both arms, MAC, TSF, and MAMC as measures of loss in muscle mass and functional physical performance were significantly correlated with malnutrition in $\mathrm{CHC}$ patients, especially those with severe liver disease. A significant decrease in HGS scores and muscle mass indices (MAC, TSF, and MAMC) were observed in severely malnourished $\mathrm{CHC}$ patients with significant fibrosis and cirrhosis; however, more reduction in HGS scores and muscle mass indices was observed in cirrhotic patients (F4) compared to those with significant fibrosis (F2-F3).
According to the HGS, previous studies found that a significant risk of malnutrition was reported in $\mathrm{CHC}$ patients. In addition to that, they concluded that HGS could provide a good alternative method in assessing a decline in protein and to be a superior indicator in detecting cirrhotic patients with malnutrition $[7,30,33]$, whereas protein depletion significantly affects HGS [84-85]. In early stages of cirrhosis, the influence on muscle strength was significantly well measured by HGS than evaluation by of MAC, TSF, and MAMC which suffer from low changes [85]. In the same manner, a significant change in HGS scores was reported compared to those of MAC, TSF, and MAMC in studied CHC patients [29, 31-36].

In this study, severity of liver disease correlated positively with adiposity, diets containing in adequate amounts of 
TABLE 4: Correlations between BMI, muscle mass scores (MAC, MAMC, and TSF), HGS, SGA malnutrition scores, and vitamin D deficiency with fibrosis markers in CHC patients with significant fibrosis and cirrhosis.

\begin{tabular}{|c|c|c|c|c|c|c|c|c|}
\hline \multirow{3}{*}{ Variables } & \multicolumn{4}{|c|}{ Significant fibrosis (mean \pm SD) } & \multicolumn{4}{|c|}{ Cirrhosis $($ mean $\pm \mathrm{SD})$} \\
\hline & \multicolumn{2}{|c|}{$\mathrm{F} 0-\mathrm{F} 1$} & \multicolumn{2}{|c|}{$\mathrm{F} 2-\mathrm{F} 4$} & \multicolumn{2}{|c|}{ F0-F3 } & \multicolumn{2}{|c|}{ F4 } \\
\hline & $r$ & $p$ & $r$ & $p$ & $r$ & $p$ & $r$ & $p$ \\
\hline BMI & 0.058 & 0.12 & 0.12 & 0.16 & 0.075 & 0.001 & 0.43 & 0.001 \\
\hline WHtR & 0.075 & 0.412 & 0.145 & 0.124 & 0.168 & 0.001 & 0.815 & 0.001 \\
\hline Vitamin $25(\mathrm{OH}) \mathrm{D}(\mathrm{ng} / \mathrm{mL})$ & 0.315 & 0.01 & 0.512 & 0.01 & 0.365 & 0.001 & 0.618 & 0.001 \\
\hline PTH (pg/mL) & 0.115 & 0.01 & 0.258 & 0.01 & 0.519 & 0.001 & 0.618 & 0.001 \\
\hline Dietary vitamin D intake (IU/d) & 0.145 & 0.01 & 0.235 & 0.01 & 0.415 & 0.001 & 0.357 & 0.001 \\
\hline Dietary Ca intake $(\mathrm{mg} / \mathrm{d})$ & 0.125 & 0.01 & 0.321 & 0.01 & 0.632 & 0.001 & 0.617 & 0.001 \\
\hline Diet score & 0.112 & 0.01 & 0.226 & 0.01 & 0.418 & 0.001 & 0.628 & 0.001 \\
\hline SAG score & -0.365 & 0.01 & -0.253 & 0.01 & -0.452 & 0.001 & -0.315 & 0.002 \\
\hline Handgrip (kg) & -0.325 & 0.001 & 0.416 & 0.001 & -0.489 & 0.001 & -0.257 & 0.001 \\
\hline MAC & -0.089 & 0.001 & 0.164 & 0.001 & -0.265 & 0.001 & -0.147 & 0.001 \\
\hline TSF & -0.032 & 0.01 & 0.187 & 0.01 & -0.163 & 0.01 & -0.129 & 0.01 \\
\hline MAMC & -0.096 & 0.001 & 0.265 & 0.001 & -0.348 & 0.001 & -0.318 & 0.001 \\
\hline
\end{tabular}

PTH: parathyroid hormone; MAC: midarm circumference in cm; TSF: triceps skin fold thickness in mm; MAMC: midarm muscle circumference in mm; CHC patients: chronic hepatitis $\mathrm{C}$ patients; SGA: subjective global assessment. Pearson's $(r)$ coefficient and $P$ values at $<0.05$ are considered statistically significant.

TABLE 5: Stepwise multiple regression analysis for malnutrition predicted by adiposity, liver fibrosis, muscle mass scores (MAC, MAMC, and TSF), HGS, SGA malnutrition scores, and vitamin D deficiency in CHC patients with significant fibrosis and cirrhosis.

\begin{tabular}{|c|c|c|c|c|}
\hline \multirow{2}{*}{ Variables } & \multicolumn{2}{|c|}{ Significant fibrosis $\left(\mathrm{F}_{2}-\mathrm{F}_{3}\right)$} & \multicolumn{2}{|c|}{ Significant cirrhosis $\left(\mathrm{F}_{4}\right)$} \\
\hline & $R 2(\beta)^{*}$ & $95 \% \mathrm{CI}$ & $R 2(\beta)^{* *}$ & $95 \% \mathrm{CI}$ \\
\hline Adiposity (BMI) & $4.6(0.35)$ & $89(75-100)$ & $5.9(0.38)$ & $86(75-98)$ \\
\hline Vitamin D deficiency & $23.8(0.42)$ & $92(82-98)$ & $16.9(0.62)$ & $81(68-96)$ \\
\hline $\mathrm{Ca}$ and vitamin $\mathrm{D}$ intake & $5.6(0.53)$ & $75(65-89)$ & $6.3(0.47)$ & $90(88-100)$ \\
\hline MAC & $2.8(0.28)$ & $84(72-92)$ & $4.3(0.32)$ & $94(88-100)$ \\
\hline MAMC & $3.6(0.22)$ & $65(55-90)$ & $5.3(0.32)$ & $78(88-100)$ \\
\hline TSF & $1.6(0.29)$ & $76(65-89)$ & $4.6(0.37)$ & $84(88-100)$ \\
\hline SGA & $3.7(0.33)$ & $79(68-92)$ & $5.8(0.48)$ & $91(82-100)$ \\
\hline HGS & $4.1(0.53)$ & $89(65-89)$ & $6.8(0.51)$ & $96(88-100)$ \\
\hline HYP $(\lg / \mathrm{mL})$ & $2.6(0.33)$ & $91(80-100)$ & $3.9(0.25)$ & $97(88-100)$ \\
\hline $\mathrm{HA}(\mathrm{ng} / \mathrm{mL})$ & $3.6(0.31)$ & $95(80-100)$ & $4.9(0.31)$ & $89(88-100)$ \\
\hline $\mathrm{FN}(\mathrm{ng} / \mathrm{mL})$ & $1.6(0.21)$ & $91(86-100)$ & $3.9(0.38)$ & $95(88-100)$ \\
\hline APRI & $2.6(0.35)$ & $98(86-100)$ & $4.5(0.41)$ & $98(85-100)$ \\
\hline Hyp index & $4.2(0.41)$ & 87 (76-98) & $5.9(0.46)$ & $90(85-100)$ \\
\hline FNPRI index & $3.2(0.52)$ & $89(72-96)$ & $4.9(0.39)$ & $89(82-100)$ \\
\hline HAPRI & $2.9(0.61)$ & $93(76-96)$ & $5.6(0.68)$ & $96(82-100)$ \\
\hline$\Sigma R 2(\%)$ & $70.5(0.38)$ & $96(76-96)$ & $89.6(0.39)$ & $98(88-100)$ \\
\hline
\end{tabular}

${ }^{*} p<0.01$ and ${ }^{* *} p<0.001 . \Sigma R 2=$ summation of cumulative values of $R$ relating to studied variables. CI: confidence interval; BMI: body mass index; MAC: midarm circumference in $\mathrm{cm}$; TSF: triceps skin fold thickness in mm; MAMC: midarm muscle circumference in mm; CHC patients: chronic hepatitis $\mathrm{C}$ patients; SGA: subjective global assessment; HYP: hydroxyproline; HA: hyaluronic acid; FN: fibronectin; APRI: AST to platelet ratio index; Hyp index: hydroxyproline to platelet ratio index; HAPRI: HA to platelet ratio index; FNPRI: FN to platelet ratio index.

vitamin $\mathrm{D}$ and Ca intake, vitamin 25(OH)D deficiency, and increased levels of PTH and negatively with SGA malnutrition scores, HGS, and muscle mass indices (MAC, TSF, and MAMC, respectively). Past studies use different parameters such as body mass index (BMI) and subjective global assessment (SGA) along with the handgrip strength (HGS) for the evaluation of malnutrition associated with liver cirrhosis [36-40].
Although liver fibronectin, hydroxyproline, hyaluronic acid, liver function, and platelet counts and their respective indices (APRI, HA index, Hyp index, and FN index) as noninvasive biomarkers were reported in evaluation of liver fibrosis [25, 26, 28, 34, 35], little is known about the importance of parameters as noninvasive biomarkers in predicating and evaluation of malnutrition associated with severe hepatic dysfunction. 
Thus, in this study, Hyp, HA, FN, APRI, HypI, HAI, and FNI as noninvasive biomarkers could be used separately or in association with vitamin D status and HGS as predictors for both liver fibrosis and malnutrition in CHC patients. Data of stepwise regression analysis revealed that adiposity, liver fibrosis (Hyp, HA, FN, APRI, HypI, HAI, and FNI), muscle mass scores (MAC, MAMC, TSF, HGS, and SGA), and vitamin $\mathrm{D}$ deficiency were associated with $\sim 70.5 \%-89.6 \%$ of the incidence of malnutrition in $\mathrm{CHC}$ patients with significant fibrosis and cirrhosis.

Previously, compared to BMI and muscle mass indices, only HGS and SGA could predict severity and short survival rates in early and late stages of cirrhotic livers [30, 36-49]. In this study, vitamin D deficiency, increased PTH, and noninvasive liver biomarkers (Hyp, HA, FN, APRI, HypI, HAI, and FNI) could be a new trend of markers measuring malnutrition associated with liver severity in CHC-patients.

\section{Conclusions}

The results showed that deficiency in vitamin D levels, HGS, SGA, and muscle mass scores (MAC, MAMC, or TSF) could be used as markers of liver pathogenicity in patients with CHC. In addition, the study concluded that noninvasive biomarkers Hyp, HA, FN, APRI, HypI, HAI, and FNI separately or in association with vitamin D status, HGS, SGA, and muscle mass scores (MAC, MAMC, or TSF) were significantly associated with an incidence of malnutrition between $70.5 \%$ and $89.6 \%$ of $\mathrm{CHC}$ patients with significant fibrosis and cirrhosis.

\section{Data Availability}

All data generated or analyzed during this study are presented in the manuscript. Please contact the corresponding author for access to data presented in this study.

\section{Conflicts of Interest}

The authors declare that they have no competing interests.

\section{Acknowledgments}

The authors are grateful to the Deanship of Scientific Research, King Saud University for funding through Vice Deanship of Scientific Research Chairs.

\section{References}

[1] J. F. Perz, G. L. Armstrong, L. A. Y. J. Farrington, Y. J. Hutin, and B. P. Bell, "The contributions of hepatitis B virus and hepatitis $\mathrm{C}$ virus infections to cirrhosis and primary liver cancer worldwide," Journal of Hepatology, vol. 45, no. 4, pp. 529538, 2006.

[2] S. Maharshi, B. C. Sharma, and S. Srivastava, "Malnutrition in cirrhosis increases morbidity and mortality," Journal of Gastroenterology and Hepatology, vol. 30, no. 10, pp. 1507-1513, 2015.
[3] F. G. Romeiro and L. Augusti, "Nutritional assessment in cirrhotic patients with hepatic encephalopathy," World Journal of Hepatology, vol. 7, no. 30, pp. 2940-2954, 2015.

[4] F. W. Ismail, R. A. Khan, L. Kamani et al., "Nutritional status in patients with hepatitis C," Journal of the College of Physicians and Surgeons-Pakistan, vol. 22, no. 3, pp. 139-142, 2012.

[5] L. Zancan, M. S. Strafella, A. Brugiolo, T. Malusa, and E. S. d'Amore, "Chronic hepatitis $\mathrm{C}$ virus infection in childhood and early cirrhosis: it is possible?," Journal of Pediatric Gastroenterology and Nutrition, vol. 30, no. 3, pp. 350-351, 2000.

[6] K. Cheung, S. S. Lee, and M. Raman, "Prevalence and mechanisms of malnutrition in patients with advanced liver disease, and nutrition management strategies," Clinical Gastroenterology and Hepatology, vol. 10, no. 2, pp. 117-125, 2012.

[7] W. Juakiem, D. M. Torres, and S. A. Harrison, "Nutrition in cirrhosis and chronic liver disease," Clinics in Liver Disease, vol. 18, no. 1, pp. 179-190, 2014.

[8] H. Y. Kim and J. W. Jang, "Sarcopenia in the prognosis of cirrhosis: going beyond the MELD score," World Journal of Gastroenterology, vol. 21, no. 25, pp. 7637-7647, 2015.

[9] Canadian guidelines for Health Care Providers, Hepatitis C: Nutrition Care, Dietitians of Canada, 2003.

[10] D. Backstedt, M. Pedersen, M. Choi, and A. Seetharam, "25Vitamin D levels in chronic hepatitis C infection: association with cirrhosis and sustained virologic response," Annals of Gastroenterology, vol. 30, no. 3, pp. 344-348, 2017.

[11] S. Abu-Mouch, Z. Fireman, J. Jarchovsky, A. R. Zeina, and N. Assy, "Vitamin D supplementation improves sustained virologic response in chronic hepatitis $C$ (genotype 1)-naïve patients," World Journal of Gastroenterology, vol. 17, no. 47, pp. 5184-5190, 2011.

[12] C. M. Lange, J. Gouttenoire, F. H. Duong, K. Morikawa, M. H. Heim, and D. Moradpour, "Vitamin D receptor and Jak-STAT signaling crosstalk results in calcitriol-mediated increase of hepatocellular response to IFN- $\alpha$," Journal of Immunology, vol. 192, no. 12, pp. 6037-6044, 2014.

[13] M. L. Yu, "Hepatitis C treatment from "response-guided" to "resource-guided" therapy in the transition era from interferon-containing to interferon-free regimens," Journal of Gastroenterology and Hepatology, vol. 32, no. 8, pp. 14361442, 2017.

[14] A. Majumdar, M. T. Kitson, and S. K. Roberts, "Systematic review: current concepts and challenges for the direct-acting antiviral era in hepatitis C cirrhosis," Alimentary Pharmacology \& Therapeutics, vol. 43, no. 12, pp. 1276-1292, 2016.

[15] N. S. Raja and K. A. Janjua, "Epidemiology of hepatitis C virus infection in Pakistan," Journal of Microbiology, Immunology, and Infection, vol. 41, pp. 4-8, 2008.

[16] P. Bedossa, D. Dargere, and V. Paradis, "Sampling variability of liver fibrosis in chronic hepatitis C," Hepatology, vol. 38, no. 6, pp. 1449-1457, 2003.

[17] G. Esmat, M. Metwally, K. R. Zalata et al., "Evaluation of serum biomarkers of fibrosis and injury in Egyptian patients with chronic hepatitis C," Journal of Hepatology, vol. 46, no. 4, pp. 620-627, 2007.

[18] L. Castera and M. Pinzani, "Non-invasive assessment of liver fibrosis: are we ready?," The Lancet, vol. 375, no. 9724, pp. 1419-1420, 2010.

[19] S. A. Gabr and A. H. Alghadir, "Prediction of fibrosis in hepatitis C patients: assessment using hydroxyproline and oxidative stress biomarkers," Virus, vol. 25, no. 1, pp. 91-100, 2014. 
[20] S. A. Gabr and A. H. Alghadir, "HCV genotypes and cellular immune response in correlation to liver fibrosis," Journal of Pure and Applied Microbiology, vol. 7, pp. 117-129, 2013.

[21] A. M. Attallah, S. O. Abdallah, A. A. Attallah et al., "Diagnostic value of fibronectin discriminant score for predicting liver fibrosis stages in chronic hepatitis C virus patients," Annals of Hepatology, vol. 12, no. 1, pp. 44-53, 2013.

[22] M. R. Álvares-da-Silva and T. Reverbel da Silveira, "Comparison between handgrip strength, subjective global assessment, and prognostic nutritional index in assessing malnutrition and predicting clinical outcome in cirrhotic outpatients," Nutrition, vol. 2, no. 2, pp. 113-117, 2005.

[23] C. B. Andreatta Gottschall, F. Feijó Nunes, M. E. Deutrich Aydos et al., "Contribution of dynamometry and the royal free hospital global assessment to the nutritional assessment of patients with chronic liver diseases," Revista Chilena de Nutricion, vol. 39, no. 4, pp. 152-158, 2012.

[24] N. J. Hackl, C. Bersch, P. Feick et al., "Circulating fibronectin isoforms predict the degree of fibrosis in chronic hepatitis C," Scandinavian Journal of Gastroenterology, vol. 45, no. 3, pp. 349-356, 2010.

[25] M. Ciocîrlan, A. R. Cazan, M. Barbu, M. Mănuc, M. Diculescu, and M. Ciocîrlan, "Subjective global assessment and handgrip strength as predictive factors in patients with liver cirrhosis," Gastroenterology Research and Practice, vol. 2017, Article ID 8348390, 2017.

[26] F. G. Romeiro and L. Augusti, "Nutritional assessment in cirrhotic patients with hepatic encephalopathy," World Journal of Hepatology, vol. 7, no. 30, pp. 2940-2954, 2015.

[27] M. Merli, M. Giusto, C. Lucidi et al., "Muscle depletion increases the risk of overt and minimal hepatic encephalopathy: results of a prospective study," Metabolic Brain Disease, vol. 28, no. 2, pp. 281-284, 2013.

[28] T. J. Cole, M. C. Bellizzi, K. M. Flegal, and W. H. Dietz, "Establishing a standard definition for child overweight and obesity worldwide: international survey," The BMJ, vol. 320, p. 1240, 2000.

[29] R. Frisancho, "New norms of upper limb fat and muscle areas for assessment of nutritional status," The American journal of clinical nutrition, vol. 34, article 2540, 1981.

[30] A. Teiusanu, M. Andrei, T. Arbanas, T. Nicolaie, and M. Diculescu, "Nutritional status in cirrhotic patients," Maedica (Buchar), vol. 7, no. 4, pp. 284-289, 2012.

[31] W. K. Seto, C. F. Lee, C. L. Lai et al., "A new model using routinely available clinical parameters to predict significant liver fibrosis in chronic hepatitis B," PLoS One, vol. 6, no. 8, article e23077, 2011.

[32] B. Farid and G. Sami, "Prediction of liver fibrosis and cirrhosis among Egyptians using noninvasive index," Journal of Pure and Applied Microbiology, vol. 1, no. 1, pp. 45-50, 2007.

[33] M. Ciocîrlan, A. R. Cazan, M. Barbu, M. Mănuc, M. Diculescu, and M. Ciocîrlan, "Subjective global assessment and handgrip strength as predictive factors in patients with liver cirrhosis," Gastroenterology Research and Practice, vol. 2017, Article ID 8348390, 2017.

[34] M. Cossio-Bolaños, C. Lee-Andruske, M. de Arruda, C. Luarte-Rocha, A. Almonacid-Fierro, and R. Gómez-Campos, "Hand grip strength and maximum peak expiratory flow: determinants of bone mineral density of adolescent students," BMC Pediatrics, vol. 18, no. 1, p. 96, 2018.

[35] L. G. Richards, B. Olson, and P. Palmiter-Thomas, "How forearm position affects grip strength," The American Journal of Occupational Therapy, vol. 50, no. 2, pp. 133-138, 1996.
[36] W. G. Dixon, M. Lunt, S. R. Pye et al., "Low grip strength is associated with bone mineral density and vertebral fracture in women," Rheumatology, vol. 44, no. 5, pp. 642-646, 2005.

[37] J. S. Harrell, R. G. Mcmurray, C. D. Baggett, M. L. Pennell, P. F. Pearce, and S. I. Bangdiwala, "Energy costs of physical activities in children and adolescents," Medicine and Science in Sports and Exercise, vol. 37, no. 2, pp. 329-336, 2005.

[38] S. G. Trost, R. R. Pate, J. F. Sallis et al., "Age and gender differences in objectively measured physical activity in youth," Medicine and Science in Sports and Exercise, vol. 34, no. 2, pp. 350355, 2002.

[39] U. Mäder, B. W. Martin, Y. Schutz, and B. Marti, "Validity of four short physical activity questionnaires in middle-aged persons," Medicine and Science in Sports and Exercise, vol. 38, no. 7, pp. 1255-1266, 2006.

[40] T. Poynard, P. Bedossa, METAVIR, and CLINIVIR cooperative study groups, "Age and platelet count: a simple index for predicting the presence of histological lesions in patients with antibodies to hepatitisC virus," Journal of Viral Hepatitis, vol. 4, no. 3, pp. 199-208, 1997.

[41] M. R. Alvares-da-Silva and T. Reverbel da Silveira, "Comparison between handgrip strength, subjective global assessment, and prognostic nutritional index in assessing malnutrition and predicting clinical outcome in cirrhotic outpatients," Nutrition, vol. 21, no. 2, pp. 113-117, 2005.

[42] W. H. El Rouby, H. A. Al Sebaee, L. N. Iskander, and M. I. Mehrez, "Nutritional assessment of patients with chronic hepatitis c treated with Sofosbuvir combined with Daclatasvir," Journal of Nursing and Health Science, vol. 6, no. 4, pp. 6167, 2017.

[43] E. Vanoirbeek, A. Krishnan, G. Eelen et al., "The anti-cancer and anti-inflammatory actions of $1,25(\mathrm{OH})_{2} \mathrm{D}_{3}$," Best Practice \& Research. Clinical Endocrinology \& Metabolism, vol. 25, no. 4, pp. 593-604, 2011.

[44] M. T. Kitson and S. K. Roberts, "Delivering the message: the importance of vitamin D status in chronic liver disease," Journal of Hepatology, vol. 57, no. 4, pp. 897-909, 2012.

[45] Y. Q. Luo, X. X. Wu, Z. X. Ling, Y. W. Cheng, L. Yuan, and C. Xiang, "Association between serum vitamin D and severity of liver fibrosis in chronic hepatitis $C$ patients: a systematic meta-analysis," Zhejing Daxue Xuebao Zirankexueban, vol. 15, pp. 900-906, 2014.

[46] S. Petta, S. Grimaudo, V. D. Marco et al., "Association of vitamin $\mathrm{D}$ serum levels and its common genetic determinants, with severity of liver fibrosis in genotype 1 chronic hepatitis C patients," Journal of Viral Hepatitis, vol. 20, no. 7, pp. 486-493, 2013.

[47] P. Lips, "Vitamin D deficiency and secondary hyperparathyroidism in the elderly: consequences for bone loss and fractures and therapeutic implications," Endocrine Reviews, vol. 22, no. 4, pp. 477-501, 2001.

[48] S. D. C. Thomas, A. G. Need, G. Tucker, P. Slobodian, P. D. O'Loughlin, and B. E. C. Nordin, "Suppression of parathyroid hormone and bone resorption by calcium carbonate and calcium citrate in postmenopausal women," Calcified Tissue International, vol. 83, no. 2, pp. 81-84, 2008.

[49] C. B. Andreatta Gottschall, F. Feijó Nunes, M. E. Deutrich Aydos et al., "Contribution of dynamometry and the royal free hospital global assessment to the nutritional assessment of patients with chronic liver diseases," Revista Chilena de Nutricion, vol. 39, no. 4, pp. 152-158, 2012. 